\title{
Producción y lectura de fan fiction en la comunidad online de la serie Fringe: transmedialidad, competencia y alfabetización mediática
}

\section{Mar Guerrero-Pico ${ }^{1}$}

Recibido: 2014-11-10

Enviado a pares: 2014-11-14
Aprobado por pares: 2014-12-05

Aceptado: 2014-12-15

DOI: 10.5294/pacla.2015.18.3.5

Para citar este artículo / To reference this article / Para citar este artigo

Guerrero Pico, M. (Septiembre de 2015). Producción y lectura de fan fiction en la comunidad online de la serie Fringe: transmedialidad, competencia y alfabetización mediática. Palabra Clave, 18(3), 722-745. DOI: 10.5294/pacla.2015.18.3.5

\section{Resumen}

Este artículo analiza los efectos de la narrativa transmedia en la creación y consumo de una clase de fanwork como el fan fiction a partir del estudio de la comunidad online de escritores y lectores dedicada a la serie de televisión Fringe (FOX, 2008-2013), una de las franquicias transmedia más relevantes de los últimos años. Basándose en un cuestionario online de tipo cualitativo y la observación participante dentro de esta comunidad, la investigación se centra en describir las características del fan fiction en el panorama transmediático y explorar de forma complementaria la adquisición de competencias interpretativas y de destrezas mediáticas por parte de los fans. El trabajo concluye revelando la tibia influencia que el conjunto de productos anexos a la serie tiene sobre el proceso creativo del fan fiction, a la vez que identifica su condición de práctica caractercéntrica, subrayando la importancia capital del personaje como el elemento sobre el que los

1 Universitat Pompeu Fabra, España. mar.guerreropico@gmail.com 
fans construyen sus expansiones narrativas. Finalmente, también se constata el valor del fan fiction como herramienta para la alfabetización mediática.

\section{Palabras clave}

Fan fiction, lectores beta, programa de televisión, alfabetización (Fuente:

Tesauro de la Unesco).

\section{Production and reading of fan fiction in the online community of the Fringe series: transmediality, competition and media literacy}

\section{Abstract}

This article analyzes the effects of transmedia narrative in the creation and consumption of a class of fan work as fan fiction based on a study of the online community of writers and readers dedicated to the TV series Fringe (FOX, 2008-2013), one of the most important transmedia franchises in recent years. Based on an online survey of qualitative and participant observation within this community, research focuses on describing the characteristics of fan fiction in the transmedia landscape and explores complementary manner interpretative skills acquisition and media skills by fans. The article concludes by revealing the warm influence that the set of associated products to the series have on the creative process of fan fiction, while identifying their condition of central character practice, stressing the importance of character as the element on which fans build their narrative expansions. Finally, the value of fan fiction as a tool for media literacy is also noted.

\section{Keywords}

Fan fiction, beta readers, television programs, literacy (source: Unesco Thesaurus). 


\section{Produção e leitura de fanfiction na comunidade on-line da série Fringe: transmidialidade, competência e alfabetização midiática}

\section{Resumo}

Este artigo analisa os efeitos da narrativa transmídia na criação e consumo de uma aula de fanwork como a fanfiction a partir do estudo da comunidade on-line de escritores e leitores dedicada à série de televisão Fringe (FOX, 2008-2013), uma das franquias transmídia mais relevantes dos últimos anos. Baseou-se em um questionário on-line de tipo qualitativo e na observação participante dentro dessa comunidade; a pesquisa centra-se em descrever as características da fanfiction no panorama transmidiático e explorar de forma complementar a aquisição de competências interpretativas e de destrezas midiáticas por parte dos fãs. $\mathrm{O}$ trabalho conclui revelando a tímida influência que o conjunto de produtos anexos à série tem sobre o processo criativo da fanfiction, uma vez que identifica sua condição de prática caractercêntrica, ao sublinhar a importância capital do personagem como o elemento sobre o qual os fãs constroem suas expansões narrativas. Finalmente, também se constata o valor da fanfiction como ferramenta para a alfabetização midiática.

\section{Palavras-chave}

Fanfiction, leitores beta, programa de televisão, alfabetização (Fonte: Tesauro da Unesco). 


\section{Introducción: narrativa transmedia y fans}

La narrativa transmedia ha cobrado especial relevancia en la agenda de los investigadores en comunicación de España y Latinoamérica en los años posteriores a la acuñación del término por Jenkins (2003). Autores como Scolari (2009, 2012, 2013a), Rodríguez Ferrándiz (2012, 2014), Vassallo de Lopes (2012), Porto-Renó et al. (2011) y Hernández-García et al. (2013) han ayudado, desde diferentes perspectivas, a abordar un concepto que puede definirse como "un proceso en el que los elementos de una ficción se dispersan a través de múltiples plataformas mediáticas con el objetivo de crear una experiencia de entretenimiento coordinada y unificada" (Jenkins, 2007).

Este proceso se caracteriza, asimismo, por constar de dos puntos de origen distinto para los elementos o expansiones que componen el universo narrativo transmedia. Por un lado, destacamos las expansiones narrativas de carácter oficial, ${ }^{2}$ aquellas desarrolladas y sancionadas por los propietarios intelectuales del relato transmedia, y, por otro, las expansiones no oficiales, los contenidos generados por los usuarios (CGU) o las audiencias receptoras de ese relato. Si bien la mayoría de los estudios sobre narrativa transmedia se han centrado hasta el momento en observar la expansión narrativa de famosas franquicias de ficción (Lost, Matrix, 24, Superman...), lo cierto es que los aportes en este campo pueden aplicarse a ámbitos de no ficción, como el periodismo, el documental (Scolari, 2013a) y la publicidad (Buckner \& Rutledge, 2011).

De la misma manera, los CGU han tenido un papel secundario en las investigaciones sobre narrativa transmedia, más interesadas en describir contenidos oficiales, entre los que destacan las redes sociales, que habilitan y alojan conversaciones entre productos mediáticos y audiencias (La-

2 A este respecto, Askwith (2007: 55-99) ofrece en su estudio sobre engagement en la televisión 2.0 una completa clasificación de ocho touchpoints o puntos de entrada, a través de los cuales el espectador se involucra en el consumo de una narrativa televisiva. Estos touchpoints son: formal program qualities (las características propias del programa), expanded access (el acceso expandido a ese mismo contenido por diferentes pantallas y plataformas), repackaged content (contenido editado o adaptado para su difusión por otros medios), ancillary content (contenido que expande la información e historias mostradas en televisión), branded products (productos de mercadotecnia), related activities (actividades relacionadas donde se incluyen, entre otros, los espacios dirigidos a fomentar los contenidos generados por el usuario), social interaction (foros de discusión y redes sociales del programa) e interaction (interacciones directas entre espectadores, aparato de televisión y contenido del programa). 
calle, 2011; Grandío \& Bonaut, 2012; Tur-Viñes \& Rodríguez Ferrándiz, 2014). Aunque disciplinas como los estudios de recepción y los fan studies han analizado durante décadas las narrativas producidas por las audiencias a partir de la interpretación de los contenidos de ficción que los fans consumen en los medios, se trata todavía de un terreno poco explorado en el contexto transmediático. Desde un punto de vista crítico, Scott ha mostrado su preocupación por que las nuevas propuestas narrativas basadas en modelos transmedia acaben por disuadir a los fans de crear sus propias interpretaciones textuales. Basándose en el despliegue transmedia en Battlestar Galactica, Scott afirma que, en el nuevo escenario, los autores o productores animan a los fans a consumir contenido autorizado que con frecuencia imita las mismas características asociadas a las narrativas producidas por los fans (rellenar los huecos de la trama, explorar universos alternativos, expandir la historia de un personaje secundario, etc.), de forma que se limita el trabajo especulativo de los aficionados y se refuerza la figura del autor (en Pearson, 2010, pp. 91-92).

Esta usurpación figurada de los productores del trabajo tradicionalmente realizado por los fans también puede ser leída en términos más amplios: la convergencia mediática y tecnológica (Jenkins, 2008), la misma que ha facilitado la explosión de universos narrativos transmedia, ha desdibujado las fronteras entre productores y fans y ha suavizado las tensiones legales que marcaron la relación entre ambas partes en el pasado. Así, más allá de las imitaciones que los autores puedan hacer de las prácticas de los fans y de las órdenes de cese por posibles delitos contra la propiedad intelectual, cada vez es más frecuente ver cómo las empresas mediáticas incorporan a los fans y sus textos dentro de sus estrategias, y al revés, este acercamiento aumenta la visibilidad y agiliza la profesionalización de los fans creadores. Pero Busse (2007) advierte del peligro que podría encerrar tal reconocimiento de las actividades de los fans: la segregación de las creaciones de los fans entre aceptables y no aceptables según la aprobación de los productores. Según Busse (2007), esto también implicaría una división entre los miembros de la propia comunidad de fans o fandom: "Ciertos grupos de fans sólo pueden ser reconocidos si siguen ciertas ideas, y si no son demasiado rebeldes ni pornográficos ni leen demasiado entrelíneas". 
Lejos de la crítica cultural, Guerrero (2012, p. 84) ha explorado las producciones de los fans ofanworks desde un enfoque que concilia semiótica narrativa y narratología para entender cómo se comportan estos textos en los sistemas transmedia. Bajo la denominación de contenidos transmediáticos generados por los usuarios (CTGU) en ficción, agrupa algunas de las modalidades creativas más extendidas, como pueden ser el fan fiction (textos escritos), el fanvid (textos audiovisuales) o el fanart (textos gráficos), y algunas otras de carácter más divulgativo, como los recaps (resúmenes escritos, audiovisuales y gráficos), los wikis (enciclopedias virtuales sobre el mundo narrativo y sus personajes) y los subtítulos en diferentes idiomas.

El presente trabajo pretende trascender el análisis textual e indagar en los posibles cambios que el modelo de narrativa transmedia ha propiciado en los modos de producción y recepción de los fanworks, concretamente del fan fiction. Para ello, se ha elegido como objeto de estudio la comunidad de escritores y lectores de fan fiction generada en torno a Fringe. Esta serie de ciencia ficción de la factoría de J. J. Abrams, emitida en la cadena estadounidense FOX entre 2008 y 2013, conforma una de las experiencias transmedia más destacables de los últimos años (Álvarez Berciano, 2012), además de contar con una activa comunidad de fans cuyo apoyo fue vital para el mantenimiento de la serie en antena durante sus dos últimas temporadas (Belsunces Gonçalves, 2011; Clarke Stuart, 2011; Cochran et al., 2014). Cómics, novelas, videocómics, juegos de realidad alternativa, páginas web ficticias y performances en eventos de audiencia masiva, videorresúmenes y guías se unen a las cinco temporadas de la serie de televisión para completar el universo narrativo que inspira las historias de los fans. En este sentido, este trabajo es un resultado de una investigación en curso sobre fan fiction basado en series de televisión en la que de forma complementaria y, haciendo uso de herramientas de análisis semionarratológicas, también se replantean las diversas estrategias narrativas (Scolari, 2013b) aplicadas en la construcción de sistemas transmedia.

\section{Objetivos y metodología}

El propósito de este estudio es ofrecer una panorámica del impacto del concepto de narrativa transmedia en los mecanismos que intervienen en la 
creación de fan fiction basado en series de televisión, así como en la lectura posterior por parte de la comunidad de fans. De forma paralela, se prestará especial atención a la posible adquisición de competencias interpretativas y la alfabetización mediática (Soep, 2012) de los fans durante el proceso de producción de fics o fanfics. ${ }^{3}$ A partir de estos objetivos, el estudio plantea tres hipótesis generales:

1. La serie de televisión (o, en su caso, el texto madre sobre el que se configura el universo narrativo transmedia) continúa siendo la única fuente utilizada para la elaboración de fics, en detrimento de otras expansiones de la franquicia.

2. El fan aumenta su conocimiento del mundo narrativo a través de la escritura y lectura de fan fiction.

3. El proceso de creación de fanfics imita en su forma más básica el proceso de edición en la industria del libro.

Desde el primer momento, la inteligencia colectiva (Lévy, 1997) de los miembros de la comunidad de fanfic de Fringe se consideró como una pieza fundamental para responder con la mayor precisión a las premisas planteadas. Basándose en otros estudios sobre las actividades de los fans en Internet (Baym, 2000; Costello \& Moore, 2007; Gray \& Mittell, 2007), se optó por una metodología de corte etnográfico que combina la elaboración de una encuesta online y la observación participante (Ardévol et al., 2003) de la autora del presente estudio como lectora involucrada en la comunidad de fanfic de la serie. Además de datos relevantes a nivel cuantitativo y cualitativo, este enfoque permite obtener una aproximación más cercana a la experiencia real, imprescindible para no incidir en generalizaciones y, al mismo tiempo, mantener la distancia necesaria para una interpretación crítica de las respuestas.

Se diseñó un cuestionario anónimo online compuesto por 38 preguntas de tipo cerrado (incluyendo tanto interrogantes de respuesta única como

3 Fic y fanfic son otros términos por los que también se conoce al fan fiction, sobre todo, para referirse a textos en concreto. 
de respuesta múltiple) y abierto. ${ }^{4}$ La encuesta contó con versiones en inglés y en español para incrementar su alcance, y se publicó una invitación para participar en ocho sitios webs de fans de Fringe. Estas webs abarcaron desde foros, ${ }^{5}$ blogs, ${ }^{6}$ comunidades de fanfic alojadas en Dreamwidth ${ }^{7}$ y Livejournal, ${ }^{8}$ hasta una cuenta de Twitter," siendo todas ellas sitios de referencia populares en este fandom por número de usuarios o miembros registrados, antigüedad y ritmo de actualización. ${ }^{10}$ El cuestionario se publicó el 26 de noviembre de 2012, coincidiendo con la emisión de la quinta y última temporada de la serie en Estados Unidos, y se mantuvo abierto hasta el 31 de enero de 2013, quince días después de la emisión del episodio final.

Tras una serie de preguntas personales (edad, sexo, lengua materna, segundo idioma), la encuesta se centraba en los hábitos de los participantes como lectores, preguntándoles por sus webs preferidas para encontrar fan fiction, el medio o plataforma para leer que utilizaban y la razón por la que leían fics de Fringe. También se propusieron preguntas para saber el grado de conformidad de los fans con la definición de canon en una franquicia transmediática como Fringe, y si eran lectores o escritores de fanfic.

En función de la respuesta a esta pregunta, la encuesta adoptaba dos itinerarios: lectores y autores. Dentro del primer bloque, se discriminaba a su vez entre lectores y lectores beta. A diferencia de los lectores comunes de fanfic, los lectores beta son aquellos fans que tienen acceso a los borradores de los fics para editarlos y sugerir cambios a los autores antes de su publicación (Karpovich, 2006). Si el participante declaraba ser sólo un lector común, se le dirigía al final de la encuesta.

4 El detalle completo del tipo de preguntas y respuestas, así como una declaración de anonimidad en el tratamiento de los datos personales, se recogen en el modelo de encuesta online utilizado en la investigación. El cuestionario en español está disponible en formato PDF en http://repositori.upf.edu/handle/10230/22963

5 http://www.fringe-forum.com. Recuperado el 8 de diciembre de 2014.

6 http://zonafringe.blogspot.com. Recuperado el 8 de diciembre de 2014.

7 http://fringetv-at-dw.dreamwidth.org. Recuperado el 8 de diciembre de 2014

8 http://fringe-olivia.livejournal.com, http://fringefiction.livejournal.com, http://fringe-tv.livejournal.com y http://cortexifans.livejournal.com. Recuperados el 8 de diciembre de 2014.

9 http://www.twitter.com/Fringenuity. Recuperado el 8 de diciembre de 2014.

10 Por ejemplo, fringe-forum.com contaba con un total de 16211 usuarios registrados, @Fringenuity alcanzaba los 3456 seguidores y comunidades como fringefiction.livejournal.com y fringetv-at-dw.dreamwidth.org contaban con 698 y 115 miembros respectivamente (datos del 8 de diciembre de 2014). Asimismo, zonafringe.blogspot.com se actualizó de forma diaria desde su creación en mayo de 2009 hasta la emisión del episodio final de la serie el 18 de enero de 2013. 
La división entre autores y lectores beta permitió confeccionar cuestiones que buscaban dar respuestas más concretas a las hipótesis de la investigación. Dentro de la secciones de lectores beta y autores, los participantes se encontraron con preguntas abiertas relacionadas, respectivamente, con la labor de betear (es decir, de revisar fics) y escribir para Fringe.

$\mathrm{Al}$ igual que los betas y los aspectos que más valoran a la hora de supervisar un borrador, los autores también tuvieron que ordenar los retos principales a los que se enfrentan cuando abordan la escritura de un fanfic de la serie, los aspectos de la serie que más resaltan en sus fics y las fuentes de inspiración general para sus historias. En este sentido, se les preguntó de forma complementaria si tenían en cuenta los contenidos extratelevisivos oficiales relacionados con Fringe cuando escribían.

Para conocer el impacto que ha tenido el fan fiction en el conocimiento del mundo narrativo de la serie, y comprobar el uso de un segundo idioma por parte de lectores beta y autores, se plantearon sendas preguntas al final de ambas secciones. A todos los participantes se les dio la oportunidad de añadir comentarios adicionales acerca de la serie y el fan fiction antes de enviar sus respuestas.

\section{Resultados}

Concluido el periodo de análisis, los resultado ${ }^{11}$ se organizaron alrededor de cuatro grandes bloques: datos generales, el fan fiction como producto transmediático, Fringe como origen de fan fiction y lectores beta y autores.

\section{Datos generales}

Un total de 304 personas respondieron a la encuesta durante las semanas que permaneció activa. El $60 \%$ son mujeres, confirmando una vez más la tendencia observada en otros trabajos sobre fan fiction y cultura de los fans (Bacon-Smith, 1992; Jenkins, 1992; Hellekson \& Busse, 2006; Hellekson, 2009): el fan fiction es una práctica con una base demográfica principalmen-

11 Un resumen de los principales resultados de la encuesta puede consultarse en: http://repositori.upf.edu/handle/10230/22963 
te femenina. La edad media de los participantes se situó en los 27,4 años. El español es la lengua materna del 63,4 \% de los participantes, mientras que un $24 \%$ se declaró anglófono. El $41 \%$ de los participantes indicó el inglés como su segundo idioma, mientras que el 12,1 \% declaró que era el español y un 6,9\%, el francés.

Con respecto a las webs que alojan fan fiction, una mayoría del 71,3\% eligió Fanfiction.net como su fuente primaria de lectura, por delante de otros sitios como ArchiveofOurOwn.com (23,1\%), LiveJournal.com (14,6 \%) y Tumblr.com $(8,2 \%) .{ }^{12}$ El ordenador continúa siendo la plataforma para leer más utilizada, con un aplastante $94,3 \%$, si bien los smartphones $(24,8 \%)$ y las tabletas $(17,4 \%)$ también alcanzaron porcentajes representativos que apuntan a una cohabitación entre las diferentes pantallas ${ }^{13}$.

Para señalar la principal razón por la que leían fan fiction de Fringe, los participantes tuvieron que escoger y clasificar sus preferencias entre una lista de ocho motivos. Los resultados dieron cuenta una vez más de la conexión emocional y afectiva que, según Hills (2002), existe entre el fan y su objeto de fandom. La necesidad de estar conectados por más tiempo a la serie y a sus personajes obtuvo la primera posición $(15,08 \%),{ }^{14}$ por delante de otras razones como la libertad de explorar nuevas relaciones entre los personajes $(13,86 \%)$, la posibilidad de descubrir nuevas teorías sobre la trama y personajes $(13,24 \%)$, leer el punto de vista de otros fans $(13,22 \%)$, distraerse $(13,13 \%)$, aprender otros idiomas $(11,31 \%)$, leer sobre algo ya conocido $(10,49 \%)$ y preferir la forma en la que los personajes son presentados en el fan fiction más que en la propia serie $(9,67 \%)$.

12 Los porcentajes representan el total de selecciones obtenido individualmente por cada fuente de lectura de fan fiction. Los participantes pudieron seleccionar más de una fuente de lectura, así, Fanfiction.net es la opción más popular, sin que ello excluya necesariamente el uso adicional de otras plataformas por parte de los participantes que eligieron Fanfiction.net.

13 Ver Nota 14.

14 Porcentaje calculado a partir de la puntuación total obtenida por cada motivo. Los motivos clasificados en posiciones superiores lograron una valoración mayor que el resto. El motor de la encuesta calculó la puntuación total sumando todas las clasificaciones ponderadas. Por ejemplo, en este caso, la necesidad de pasar más tiempo conectados a la serie obtuvo el primer lugar, acumulando un total de 844 puntos, que se traducen en un porcentaje del 15,08 \%. Todos los resultados desprendidos de preguntas en las que los participantes hubieron de elegir y ordenar sus preferencias entre una lista de elementos figuran como porcentajes a lo largo del presente texto. 


\section{El fan fiction como producto transmediático}

Aunque los CTGU son una parte indisociable de las expansiones narrativas oficiales cuando se aborda la idea de transmedialidad, cabe cuestionarse si los fans son igualmente receptivos a considerar esas expansiones dispersas en distintos soportes cuando escriben sus obras de fan fiction. El análisis arrojó una respuesta razonablemente negativa a esta pregunta, ya que el 54,6\% de los autores afirmaron basar sus historias sólo en la serie de televisión de entre el abanico de contenidos que ofrece una franquicia transmedia como Fringe, y el 11,4 \% indicó no ser consciente de la existencia de tales contenidos. No obstante, un porcentaje del 34,1\% sí tiene en cuenta otras expansiones narrativas, al considerarlas parte del canon.

La definición de canon (ver tabla 1) también alumbró posturas resistentes a las implicaciones del concepto de narrativa transmedia, al no haber un consenso claro entre los participantes acerca de qué elementos del mundo narrativo componen dicho canon. Si bien la consideración única de la serie de televisión como fuente canónica es la menos popular de las tres opciones dadas, la cifra sólo está tres puntos por debajo de la segunda de las opciones "benévolas" con los materiales derivados. Dentro de éstas, la opción que incorpora a los productores y guionistas de la serie como figuras sancionadoras es la preferida, reconociendo la autoridad última que aquellos tienen sobre el mundo narrativo. A la vista de estos datos, puede decirse que el actual panorama transmediático ha intensificado el tradicional debate entre los fans acerca de la naturaleza del canon. Hasta cierto punto, también ha reforzado la necesidad de los fans de apoyarse en la palabra de los creadores para orientar sus opiniones, sobre todo en los casos de sistemas transmedia de origen televisivo cuya expansión se ha llevado a cabo de forma desorganizada (Scolari et al., 2012), y en los que los fans han detectado errores de continuidad entre las diferentes expansiones.

Al pedirles que priorizaran los elementos en los que más se fijan cuando realizan sus tareas, lectores beta y autores coincidieron en destacar la caracterización fiel de los personajes con respecto al canon como el aspecto más importante. En concreto, para los autores los tres retos principales son, en este orden: mantener la caracterización original independientemente de 


\section{Tabla 1 \\ ¿Qué es una fuente canónica en Fringe?}

\begin{tabular}{|l|c|}
\hline \multicolumn{1}{|c|}{ Definición } & Porcentaje (n=209) \\
\hline Sólo la serie de televisión. & $29,70 \%$ \\
\hline $\begin{array}{l}\text { La serie de televisión y otros materiales derivados (por ejemplo: los cómics y videocómics } \\
\text { de Fringe) o información de contexto, sólo si los productores o creadores los consideran } \\
\text { parte de la historia. }\end{array}$ & $37,30 \%$ \\
\hline $\begin{array}{l}\text { La serie de televisión y otros materiales derivados (por ejemplo: los cómics y videocómics } \\
\text { de Fringe) que expandan la historia siempre que sean oficiales. No hace falta la palabra de los } \\
\text { productores para considerarlos canon. }\end{array}$ & $31,6 \%$ \\
\hline Otros. & $1,40 \%$ \\
\hline
\end{tabular}

Fuente: elaboración propia.

si el fanfic respeta o no el canon de la serie $(11,23 \%)$, mantener el ritmo de la historia $(10,41 \%)$ y saber transmitir las emociones de los personajes a través de los diálogos $(10,37 \%) .{ }^{15}$ Si bien para los lectores beta los diálogos ocupan la primera posición (11,42\%), seguidos de la caracterización $(11,07 \%)$ y la ortografía y gramática $(10,25 \%),{ }^{16}$ las respuestas a las preguntas abiertas apuntalaron el tratamiento de los personajes como un punto crítico para considerar la buena calidad de un fic, en general:

Creo que los personajes deben estar bien caracterizados y, si no lo están, debe haber una buena razón para ello. En el caso de Fringe, esto podría ser una versión alternativa de un personaje 0 un viaje en el tiempo que cambiara algún aspecto que repercutiese en su formación, por ejemplo, ¿qué pasaría si el padrastro de Olivia nunca la hubiera maltratado? En general, los personajes deben estar bien caracterizados y el mejor fan fiction es aquel cuyos diálogos reflejan el ritmo de los diálogos de la serie ${ }^{17}$ (mujer, 30 años, Estados Unidos, acceso al cuestionario el 28 de noviembre de 2012 desde fringefiction.livejournal.com].

15 Otros retos para los autores son, en este orden de preferencia: construir tramas largas (9,26\%), encontrar el tono adecuado $(8,76 \%)$, describir la atmósfera del fic $(7,16 \%)$, no equivocarse en la información científica $(6,91 \%)$, encontrar una premisa original para escribir un fic (6,22\%), mantenerse fiel al canon y al género $(6,09 \%)$, escribir escenas eróticas $(5,92 \%)$, tratar temas polémicos sin caer en lo superficial $(4,61 \%)$, no cometer errores ortográficos $(4,62 \%)$, documentarse antes de escribir (4,20\%), escribir escenas ligeras o de humor $(2,30 \%)$, ser fiel al universo narrativo propuesto por otro fan autor $(1,94 \%)$.

16 Otros aspectos tenidos en cuenta por los lectores beta son, en este orden: originalidad (9,42\%), ritmo (9,25\%), fidelidad al canon $(8,97 \%)$, calidad de las descripciones $(8,92 \%)$, realismo $(7,64 \%)$ precisión en los datos científicos $(7,63 \%)$, complejidad de la trama $(6,49 \%)$, fidelidad al universo narrativo propuesto por otro fan autor $(4,48 \%)$, atrevimiento $(4,46 \%)$.

17 Traducción propia del original en inglés. 
Este interés en preservar la voz de los personajes por encima de otras cuestiones (como la fidelidad al canon y al género en general, la originalidad, y el ser rigurosos en la presentación de datos científicos en el caso específico de Fringe) da cuenta de dos fenómenos. En primer lugar, el fan fiction como CTGU está fuertemente determinado por el canon del que se deriva; tanto autores como lectores no renuncian del todo a buscar la representación del material original en los textos. La popularidad de los fics de universo alternativo (comúnmente denominados por su acrónimo en inglés, $A U$, de alternate universe), subgénero de fan fiction donde se emplaza a los personajes en espacios y tiempos distintos a los de la serie, o se modifican hechos de la trama, es una prueba de que esta representación se articula fundamentalmente en el nivel de los personajes más que en la historia o el mundo narrativo.

Tal particularidad permite introducir el segundo fenómeno del fan fiction como CTGU, su condición de práctica caractercéntrica, donde el personaje es el puente que une la expansión narrativa creada por el fan escritor con el universo canónico en el que se inspira. Esta importancia capital que en el texto del fan adquiere el personaje como perpetuador de la narrativa confirma el punto de vista desarrollado por Bertetti (2014) en su estudio de algunas franquicias transmedia de la primera mitad del siglo XX (como Conan el Bárbaro). Bertetti sitúa a los personajes en el origen de la construcción de esas franquicias, y añade: "La presencia del mismo personaje en diferentes textos y en distintas plataformas mediáticas $[. .$.$] no im-$ plica necesariamente que estos textos o plataformas compartan el mismo mundo" (2014, p. 2346). De nuevo, no es difícil pensar en esta afirmación mientras se lee un fic de universo alternativo.

\section{Fringe como origen de fan fiction}

El número de participantes en la encuesta da una idea del reducido tamaño de la comunidad de fanfic dedicada a Fringe, tal y como se confirmó en una de las respuestas dadas:

Es una comunidad pequeña. Pienso que la calidad y la coherencia que ha demostrado la serie durante todos estos años, dándonos mucho más de lo que los fans esperábamos, ha contribuido a que los fanfics 
no sean, a mi juicio, tan numerosos como en otros fandoms (por ejemplo, Expediente $X$ ) donde el fan tenía que rellenar los huecos que el canon iba dejando (mujer, 42 años, España, acceso al cuestionario el 27 de noviembre de 2012 desde zonafringe.blogspot.com).

Los fans establecen una relación directa entre la cantidad de fanfic producido dentro del propio fandom y la calidad del material de origen. De esta forma, una historia cuyo relato cuente con numerosas incongruencias, o deje tramas o aspectos sin resolver de forma satisfactoria, es más proclive a ser objeto de múltiples reescrituras que, a su vez, no nieguen el canon. Al mismo tiempo, existe una relación entre las características del texto madre y el fan fiction al que da lugar en tanto que el fan justifica sus propias decisiones creativas basándose en lo propuesto por el canon:

\begin{abstract}
Una gran parte de mi placer escribiendo fanfics viene de poder ser capaz de construir mi propio fanon ${ }^{18}$ en paralelo a la serie donde todo encaja perfectamente dentro del canon. Pero no puedo hacer eso con Fringe porque todavía no ha terminado; quiero saber todo lo que pueda de la historia antes de lanzarme a la aventura. Antes ya había conseguido escribir bastante fan fiction sobre Heroes cuando estaba en emisión, pero eso sólo fue posible porque el canon de la serie no paraba de contradecirse cada dos por tres (y los guionistas se inventaban salidas de lo más rebuscadas), así que tenía sentido que el fanfic también fuera algo parecido ${ }^{19}$ (hombre, 23 años, Singapur, acceso al cuestionario el 29 de noviembre de 2012 desde fringe-tv. livejournal.com).
\end{abstract}

Si bien Fringe se presenta bajo el sello de una serie de ciencia ficción impulsada por el clásico arco argumental de una gran conspiración que amenaza a toda la humanidad, la subtrama de los personajes cobra similar importancia al insistir en las consecuencias que para el personaje de Walter Bishop tiene el hecho de haber secuestrado al doble de su hijo en la realidad alternativa. La condición general del fan fiction como práctica caractercéntrica fomenta la creación de historias con temáticas enfocadas a explorar las motivaciones e interacciones de los protagonistas de la serie (ver tabla 2).

18 Busse y Hellekson (2006, p. 9) definen el fanon como "los eventos creados por la comunidad de fans dentro de un fandom y que se van propagando de texto a texto. A menudo el fanon crea detalles o lecturas particulares de un personaje aunque no estén avaladas por el canon".

19 Traducción propia del original en inglés. 
Al mismo tiempo, la elección de los géneros de fan fiction apunta a realzar ese factor humano, primando los textos de daño/consuelo, ${ }^{20}$ de romance y los estudios de personaje, sobre todo, de personajes que no han disfrutado de demasiado tiempo en pantalla.

\section{Tabla 2 \\ ¿En cuál de los siguientes aspectos de la serie te concentras cuando escribes tus historias?}

\begin{tabular}{|l|c|c|}
\hline \multicolumn{1}{|c|}{ Tema } & Porcentaje & Posición \\
\hline Relaciones familiares complejas & $14,50 \%$ & 1 \\
\hline Relaciones amorosas & $12,76 \%$ & 2 \\
\hline Compañerismo y dinámicas de equipo & $10,16 \%$ & 3 \\
\hline Ciencia y eventos Fringe & $9,03 \%$ & 4 \\
\hline Redención y perdón & $8,60 \%$ & 5 \\
\hline Amistad & $8,35 \%$ & 6 \\
\hline Subtexto & $5,54 \%$ & 7 \\
\hline Soledad & $4,45 \%$ & 8 \\
\hline Conspiraciones y terrorismo mundial & $4,41 \%$ & 9 \\
\hline Problemas de confianza & $4,31 \%$ & 10 \\
\hline Límites éticos en la ciencia & $4,20 \%$ & 11 \\
\hline Ciencia vs. fe & $3,86 \%$ & 12 \\
\hline Consumo de drogas & $3,39 \%$ & 13 \\
\hline Igualdad de la mujer en el trabajo & $3,28 \%$ & 14 \\
\hline El científico loco & $3,16 \%$ & 15 \\
\hline
\end{tabular}

Fuente: elaboración propia.

Que Fringe incluya los universos alternativos dentro de su mitología hace que la experiencia de escribir $A U$ fics sea diferente para los fans de la serie. La creación de fan fiction de universo alternativo dentro de esta comunidad se contempla como un medio en que el mundo posible imaginado por el fan puede quedar legitimado por los sucesos acaecidos en la serie de televisión, además del propio juego especulativo que supone la escritura de este tipo de fan fiction.

20 Daño/consuelo (hurt/comfort o $h / c$, en inglés) es un subgénero de fanfic en el que un personaje es herido o sufre por algún motivo y otro se encarga de consolarlo y ofrecerle apoyo. 


\section{Lectores beta y autores}

Una de las cuestiones más interesantes alumbradas por la encuesta es la consideración de los lectores beta como una suerte de guardianes encargados de velar por la representación canónica de los personajes en el fan fiction. Si los aspectos en los que los betas centran su labor están directamente relacionados con el canon, no sorprende que entre las cualidades exigidas al beta modelo se encuentren poseer un gran conocimiento del mundo narrativo y ser estricto con los detalles del mismo, además de contar con una ortografía impoluta. Estas características apoyan y dan sentido a otras, como las necesidades del autor y la obligación de adaptarse a ellas, hacer sugerencias y críticas constructivas para mejorar el texto, o ayudar al autor en caso de bloqueo creativo:

Tengo tres betas, y les consulto dependiendo del fandom para el que esté escribiendo [...]. Llevo escribiendo 20 años, de forma profesional y por puro entretenimiento, y siempre, SIEMPRE (sic) saco algo de mis conversaciones con ellos. Puede ser algún pequeño matiz sobre el personaje que no se había plasmado en el texto, y que sólo estaba en mi cabeza, pero que resulta ser importante para entender mi punto de vista; 0 una idea genial que lleva a otro nivel una historia que me estaba costando escribir hasta ese momento... Pero siempre es algo ${ }^{21}$ (Mujer, 30 años, Australia, acceso al cuestionario el 28 de noviembre de 2012 desde cortexifans.livejournal.com).

Lectores beta y autores coinciden en equiparar su relación con la del editor-escritor que se observa en el ámbito literario, aunque insisten en recalcar dos diferencias fundamentales. En primer lugar, se trata de un intercambio altruista en el que primarían los valores sociales, de construcción de una comunidad, sobre los económicos, una situación que tradicionalmente se asocia a la esfera de lo femenino (Hellekson, 2009), el género predominante en las comunidades de fan fiction. En segundo lugar, y por esta misma razón, se trata de un vínculo que carece de la jerarquía de poder típica entre un editor-escritor profesionales, dando lugar a una dinámica entre pares, donde el beta-editor tiene, en última instancia, una capacidad

21 Traducción propia del original en inglés. 
de decisión mucho más limitada. Algunos lectores beta reconocieron haber forjado amistades con autores a través de esta actividad y hablan de las ventajas que ello supone:

\begin{abstract}
Creo que debe existir cierto nivel de confianza. He hecho varios amigos: de hecho, mi mejor amiga es una mujer que beteó mi fic, y yo el suyo. Nos hicimos socias de escritura y hasta hemos coescrito un par de cosas. Sacamos ideas de forma conjunta, nos damos ánimos cuando estamos bloqueadas y somos brutalmente honestas con la forma de escribir de la otra ${ }^{22}$ (mujer, 54 años, Estados Unidos, acceso al cuestionario el 02 de diciembre de 2012 desde @Fringenuity).
\end{abstract}

La estrecha colaboración que se puede llegar a establecer entre betas y autores pone en contexto el proceso de ideación de los fanfics. Así, los fans eligieron las conversaciones con otros compañeros dentro de las comunidades como la principal fuente de inspiración para sus historias $(26,74 \%)$, seguidas de la música $(24,67 \%)$, otras historias procedentes de la literatura, la cultura popular o la historia $(21,49 \%)$, la propia vida personal del autor $(17,76 \%)$, y el arte $(9,34 \%)$.

Betas y autores perciben su labor como una actividad en continua evolución. En este escenario, no es extraño que el proceso creativo facilite la adquisición de diversas destrezas mediáticas, sobre todo en el caso de los autores. Algunos de ellos no ocultaron en sus respuestas que la práctica del fan fiction les estaba ayudando "inmensamente" 23 a mejorar su capacidad literaria con vistas a convertirse en autores profesionales algún día, ya sea por las indicaciones de sus betas como por los comentarios que los lectores dejan al final de cada capítulo publicado.

Con respecto a las competencias interpretativas adquiridas en relación con Fringe, un 71,7 \% de los autores afirmaron tener un mayor conocimiento del mundo narrativo y haber mejorado su experiencia de visionado de la serie gracias al fan fiction. Esto presenta una paradoja si tenemos en cuenta el relativamente escaso uso de las expansiones transmedia oficiales para escribir fics y la división de opiniones acerca de la canonicidad de las

22 Traducción propia del original en inglés.

23 Hombre, 19 años, Canadá, acceso al cuestionario el 26 de noviembre de 2012 desde fringe-forum.com. 
mismas. De este modo, se pondría en duda el binomio que asocia un mayor consumo de expansiones oficiales con un mayor grado de competencias interpretativas (Scolari, 2009), y se abriría un interrogante hacia un modelo de adquisición en donde vías alternas impulsadas por lógicas bottom-up (como los CTGU) convivan en paralelo con las estructuras top-down dentro del contexto de la narrativa transmedia.

\section{Conclusiones}

Se puede afirmar que el impacto del desarrollo transmedia del texto madre en la creación de fan fiction es todavía mínimo a la luz de los resultados del presente estudio sobre una franquicia como Fringe. Aunque los fans reconocen la existencia de expansiones oficiales que amplían el universo narrativo, al mismo tiempo cuestionan su legitimidad y no terminan de incorporarlas de forma efectiva a sus procesos de escritura, siendo la serie de televisión la única fuente válida. La metodología utilizada no ha permitido responder a la causa concreta de esta aparente falta de interés. Por ello, sería pertinente seguir trabajando en esta línea de investigación y perfilando las herramientas de análisis para tratar de despejar futuras incógnitas, comola de una posible correlación entre la calidad de las expansiones transmedia y la mayor o menor abundancia de fics que las toman como base.

Asimismo, y como ya hemos adelantado, esta situación obliga a revisar ideas preestablecidas acerca de cómo se articula la adquisición de competencias interpretativas en los sistemas transmedia. Mientras que las expansiones oficiales de Fringe estriban en la expansión de grandes universos narrativos, guiando al fan desde dentro hacia afuera, el fan fiction como expansión no oficial es una práctica caractercéntrica, donde la construcción de sentido tiende a ser centrípeta, introspectiva, ahondando en las motivaciones de personajes y pasando por las del propio fan autor: "El fan fiction no sólo es un reflejo de la serie, sino de la gente que la ve y escribe sobre ella”, argumenta una fan. ${ }^{24}$ Esta propuesta, lejos de presentar una oposición entre dos formas distintas de adquirir competencias, aboga por dos modos complementarios que serían ejemplos de la profundidad y variedad que

24 Mujer, 54 años, Estados Unidos, acceso al cuestionario el 2 de diciembre de 2012 desde @Fringenuity. 
puede alcanzar una narrativa transmedia en toda su amplitud. Así, esta visión insta a seguir explorando taxonomías de expansiones transmedia que reflejen esta complejidad de forma integrada, conjugando expansiones oficiales y no oficiales.

Este trabajo también ha permitido confirmar al fan fiction como un vivero para la alfabetización mediática, una cualidad aplicable a otros ejemplos de CTGU tanto de ficción (hacer un fan vid requiere saber editar imagen y sonido) como de no ficción (una noticia generada por el lector de un diario online supone conocimientos básicos de redacción periodística). De forma adicional, se ha podido comprobar el potencial específico del fan fiction como vehículo para el aprendizaje del inglés como segundo idioma (Black, 2005), si comparamos el alto porcentaje de hispanohablantes en el estudio con el relato de más de un lector que lamenta "los pocos escritores en español que escriben fanfic de calidad" ${ }^{25}$ y, especialmente, de autores que confesaron "no ser capaces" ${ }^{26}$ de escribir en español por haberse acostumbrado a crear en inglés, por coherencia con el tono original de Fringe, o por llegar a más lectores. En este sentido, futuros estudios sobre escritura y lectura de fan fiction, en tanto herramienta educativa para fomentar el aprendizaje de competencias mediáticas e idiomáticas en las escuelas, podrían ayudar a concretar más las posibilidades que ofrece la práctica de este CGTU más allá de las fronteras de su fandom natural.

\section{Referencias}

Álvarez Berciano, R. (2012). Tensiones de la narrativa serial en el nuevo sistema mediático. Anàlisi Monográfic: Audiovisual 2.0, 33-47. Recuperado el 10 de agosto de 2014 de http://www.analisi.cat/index. $\mathrm{php} /$ analisi/article/view/m2012-alvarez/m2012-alvarez

Ardèvol, E., Bertrán, M., Callén, B. \& Pérez, C. (2003). Etnografía virtualizada: la observación participante y la entrevista en semiestructu-

25 Mujer, 45 años, España, acceso al cuestionario el 26 de noviembre de 2012 desde zonafringe.blogspot.com.

26 Mujer, 23 años, Argentina, acceso al cuestionario el 29 de noviembre de 2012 desde fringe-olivia.livejournal.com. 
rada en línea. Atenea Digital, 3, 72-92. Recuperado el 15 de agosto de 2014 de http://www.redalyc.org/articulo.oa?id=53700305

Askwith, I. (2007). Television 2.0: Reconceptualizing TV as an Engagement Medium. Tesis de Máster, Massachusetts Institute of Technology, EE. UU. Recuperado el 8 de diciembre de 2014 de http://cms. mit.edu/research/theses/IvanAskwith2007.pdf

Bacon-Smith, C. (1992). Enterprising women: Television fandom and the creation of popular myth. Filadelfia: University of Pennsylvania Press.

Baym, N. K. (2000). Tune in, log on: Soaps, fandom and online community. Thousand Oaks: Sage Publications.

Belsunces Gonçalves, A. (2011). Producción, consume y prácticas culturales en torno a los nuevos media en la cultura de convergencia: el caso de Fringe como narración transmedia y lúdica. Tesis de Máster, Universitat Oberta de Catalunya, España.

Bertetti, P. (2014). Toward a Typology of Transmedia Characters. International Journal of Communication, 8, 2344-2361. Recuperado el 17 de agosto de 2014 de http://ijoc.org/index.php/ijoc/article/ view/2597/1201

Black, R. W. (2005). Access and affiliation: The literacy and composition practices of English-language learners in an online community. Journal of Adolescent \& Adult Literacy, 49(2), 118-128. DOI: http://dx.doi.org/10.1598/JAAL.49.2.4

Buckner, B. \& Rutledge, P. (2011). Transmedia storytelling for marketing and branding: It is not Entertaiment. It is survival. International Marketing Association. Recuperado el 8 de diciembre de 2014 de http: / / athinklab.com/wp-content/uploads/2011/04/2011-03-IMA-Published-Piece-TS.pdf 
Busse, K. (2007). Podcasts and the fan experience of disseminated media commentary. Ephemeral Traces. Recuperado el 15 de agosto de 2014 de http://kbusse.wordpress.com/2007/05/01/my-presentation-for-flow-2006/

Busse, K. \& Hellekson, K. (2006). Introduction. En Hellekson, K. \& Busse, K. (Eds.), Fan Fiction and Fan Communities in the Age of the Internet: New essays (pp. 5-32). Jefferson, NC: McFarland.

Clarke Stuart, S. (2011). Into the looking glass: Exploring the worlds of Fringe. Toronto: ECW Press.

Cochran, .T, Ginn, S. \& Zinder, T. (Eds.). (2014). The multiple worlds of Fringe: Essays on the J. J. Abrams Science Fiction Series. Jefferson, NC: McFarland.

Costello, V. \& Moore, B. (2007). Cultural outlaws: An examination of audience activity and online television fandom. Television \& New Media, 8(2), 281-143. DOI: http://dx.doi. org/10.1177/1527476406299112

Gray, J. \& Mittell, J. (2007). Speculation on Spoilers: Lost Fandom, Narrative Consumption and Rethinking Textuality. Participations, 4(1). Recuperado el 15 de agosto de 2014 de http:/ / www.participations. org/Volume\%204/Issue\%201/4_01_graymittell.htm

Grandío, M. M. \& Bonaut, J. (2012). Transmedia audiences and television fiction: A comparative approach between Skins (UK) and El Barco (Spain). Participations, 9(2), 558-574. Recuperado el 8 de diciembre de 2014 de http:/ / www.participations.org/Volume\%209/ Issue $\% 202 / 30 \% 20 \mathrm{Grandio} \% 20 \mathrm{Bonaut}$.pdf

Guerrero, M. (2012). La red enmarañada: narrativa transmedia en ficción femenina. Signo y Pensamiento, 31(61), 74-90. Recuperado el 10 de agosto de 2014 de http: / / revistas.javeriana.edu.co/index.php/ signoypensamiento/article/view/4410/3325 
Hellekson, K. (2009). A fannish field of value: Online fan gift culture. Cinema Journal, 48(4), 113-118. DOI: http://dx.doi.org/10.1353/ cj.0.0140

Hernández-García, P., Ruiz-Muñoz, M. J. \& Simelio Solá, N. (2013). Propuesta metodológica para el análisis de la ficción televisiva 2.0. Palabra Clave, 16(2), 449-469. Recuperado el 8 de diciembre de 2014 de http://palabraclave.unisabana.edu.co/index.php/palabraclave/article/view/2588

Hills, M. (2002). Fan cultures. Londres: Routledge.

Jenkins, H. (1992). Textual poachers: Television fans and participatory culture. Londres: Routledge.

Jenkins, H. (2003). Transmedia storytelling. Moving characters from books to films to video games can make them stronger and more compelling. Technology Review. Recuperado el 10 de agosto de 2014 de http://www.technologyreview.com/news/401760/transmedia-storytelling/

Jenkins, H. (2007). Transmedia Storytelling 101. Confessions of an Aca-Fan: The Oficial Weblog of Henry Jenkins. Recuperado el 10 de agosto de 2014 de http://henryjenkins.org/2007/03/transmedia_storytelling_101.html

Jenkins, H. (2008). Convergence culture: la cultura de la convergencia de los medios de comunicación. Barcelona: Paidós.

Karpovich, A. I. (2006). The audience as editor: The role of beta readers in online fan fiction communities. En K. Hellekson \& K. Busse (Eds.), Fan fiction and fan communities in the age of the Internet: New essays (pp. 171-188). Jefferson, NC: McFarland. 
Lacalle, R. (2011). La ficción interactiva: televisión y web 2.0. Ámbitos, 20, 87-107. Recuperado el 8 de diciembre de 2014 de http:/ / www.redalyc.org/pdf/168/16821321005.pdf

Lévy, P. (1997). Collective intelligence: Mankind's emerging world in cyberspace. Nueva York: Plenum Press.

Pearson, R. (2010). Fandom in the Digital Era. Popular Communication: The Internacional Jornal of Media and Culture, 8(1), 84-95. DOI: http://dx.doi.org/10.1080/15405700903502346.

Porto-Renó, D., Versuti, A. C., Moraes-Gonçalves, E. \& Gonsciola, V. (2011). Narrativas transmídia: diversidade social, discursiva y comunicacional. Palabra Clave, 14(2), 201-215. Recuperado el 20 de octubre de 2014 de http://palabraclave.unisabana.edu.co/index. php/palabraclave/article/view/1973/2521

Rodríguez Ferrándiz, R. (2012). Sangre fresca publicitaria: True Blood y las transfusiones de la ficción. Anàlisi Monográfic: Audiovisual 2.0, 65-80. Recuperado el 10 de agosto de 2014 de http:/ / www.analisi.cat/index.php/analisi/article/view/m2012-rodriguez/m2012-rodriguez

Rodríguez Ferrándiz, R. (2014). El relato por otros medios: ¿un giro transmediático? CIC, Cuadernos de Información y Comunicación, 19, 1937. Recuperado el 8 de diciembre de 2014 de http: / / revistas.ucm. es/index.php/CIYC/article/view/43901/41499

Scolari, C. A. (2009). Transmedia storytelling: Implicit consumers, narrative worlds, and branding in contemporary media production. International Journal of Communication, 3, 586-606. Recuperado el 10 de agosto de 2014 de http://ijoc.org/index.php/ijoc/article/ view $/ 477 / 336$

Scolari, C. A., Jiménez, M. \& Guerrero, M. (2012). Narrativas transmediáticas en España: cuatro ficciones en busca de un destino cross-media. Comunicación y Sociedad, 25(1), 137-164. 
Scolari, C.A. (2013a). Narrativas transmedia: Cuando todos los medios cuentan. Barcelona: Deusto.

Scolari, C. A. (2013b). Lostology: Transmedia storytelling and expansion/ compression strategies. Semiotica, 193, 1-24. DOI: http://dx.doi. org/10.1515/sem-2013-0038

Soep, E. (2012). Generación y recreación de contenidos digitales por los jóvenes: implicaciones para la alfabetización mediática. Comunicar, 19(38), 93-100. DOI: http://dx.doi.org/10.3916/C38-2012$02-10$

Tur-Viñes, V. \& Rodríguez Ferrándiz, R. (2014). Transmedialidad: series de ficción y redes sociales. El caso de Pulseras Rojas en el grupo oficial de Facebook (Antena 3, España). Cuadernos.info, 34, 115131. Recuperado el 8 de diciembre de 2014 de http: / / cuadernos. uc.cl/uc/index.php/CDI/article/view/549

Vassallo de Lopes, M. I. (2012). A case study on transmedia reception: Fandom on Facebook and social issues in the Brazilian telenovela Passione. Anàlisi Monográfic: Audiovisual 2.0, 111-132. Recuperado el 10 de agosto de 2014 de http://journals.uoc.edu/index.php/ analisi/article/view/m2012-vassallo/m2012-vasallo 